\title{
Liver toxicity during temozolomide chemotherapy caused by Chinese herbs
}

\author{
Thomas Melchardt', Teresa Magnes ${ }^{1}$, Lukas Weiss ${ }^{1}$, Michael Grundbichler ${ }^{1}$, Michael Strasser ${ }^{2}$, Clemens Hufnagl ${ }^{1}$, \\ Martin Moik', Richard Greil ${ }^{1}$ and Alexander Egle ${ }^{1^{*}}$
}

\begin{abstract}
Background: Complementary and alternative medicine is often used by patients with malignant glioma. Although several interactions of various alternative agents with chemotherapy are known, none has been described for temozolomide so far.

Case presentation: We report the case of severe liver toxicity with jaundice during radiochemotherapy with temozolomide likely due to interaction with a popular Chinese herbal formula after surgery for glioblastoma. After cessation of the herbal formula as well as the chemotherapy liver enzymes slowly normalized. Due to tumor progression the patient was retreated with temozolomide for 5 cycles without toxicity. Because of further progression combination treatment of bevacizumab and irinotecan was started and again no liver toxicity was observed.

Conclusions: We conclude that the observed toxicity with jaundice was probably caused by an interaction of this popular Chinese formula and temozolomide. This is the first report about a relevant interaction of temozolomide and any herbal formula.
\end{abstract}

Keywords: Temozolomide, Chinese herbs, Interaction, Liver toxicity, Hepatitis

\section{Background}

Complementary and alternative medicine (CAM) is frequently used by glioblastoma patients [1] but treating physicians are often left uninformed about its use. These patients are potentially at increased risk for herb-drug interactions resulting in increased toxicity due to higher serum levels of cytostatics on the one hand or undertreatment due to decreased efficacy caused by lower serum levels on the other hand.

\section{Case presentation}

A 56 year old female Caucasian patient was diagnosed with a temporal-parietal glioblastoma WHO $\mathrm{IV}^{\circ}$ after further evaluation for cephalea. Complete resection was performed using 5-aminolevulinic acid guided surgery and postoperative magnetic resonance investigation showed a small rest tumor. The patient had a Karnofsky performance status

\footnotetext{
* Correspondence: a.egle@salk.at

'Department of Internal Medicine III, Salzburg Cancer Research Institute, Paracelsus Medical University Salzburg, Austria, Müllner-Hauptstrasse 48, Salzburg 5020, Austria

Full list of author information is available at the end of the article
}

scale of $90 \%$ after resection without neurological deficiencies and was referred to our department for further treatment with radiotherapy and temozolomide (TEM). Co-medication consisted of dexamethason $4 \mathrm{mg}$ per day because of edema, pantoprazol, levetiracetam and mirtazapine because of depression. Further medical history consisted of hypertension and recurrent cutaneous herpes infections. Adjuvant concomitant radiochemotherapy (RCT) was started with an absolute dose of $140 \mathrm{mg}$ TEM per day 4 weeks after primary resection. Furthermore, prophylactic valaciclovir was prescribed. Treatment was initially well tolerated and the steroid was tampered. Unfortunately, TEM had to be stopped after 32 days of treatment and 24 fractions of radiotherapy because of grade $\mathrm{II}^{\circ}$ thrombopenia and increasing liver enzymes. Mirtazapine, valaciclovir and pantoprazol were paused and radiotherapy was completed as planned after 30 fractions. Because of further increasing liver enzymes and jaundice (bilirubin: $16.7 \mathrm{mg} / \mathrm{dl}$; AST: $263 \mathrm{U} / \mathrm{l}$; ALT: $585 \mathrm{U} / \mathrm{l}$ ) the patient was hospitalized and underwent further evaluation including exclusion of viral hepatitis, Epstein-Barr virus or cytomegalovirus-infection, computed tomography scan as 


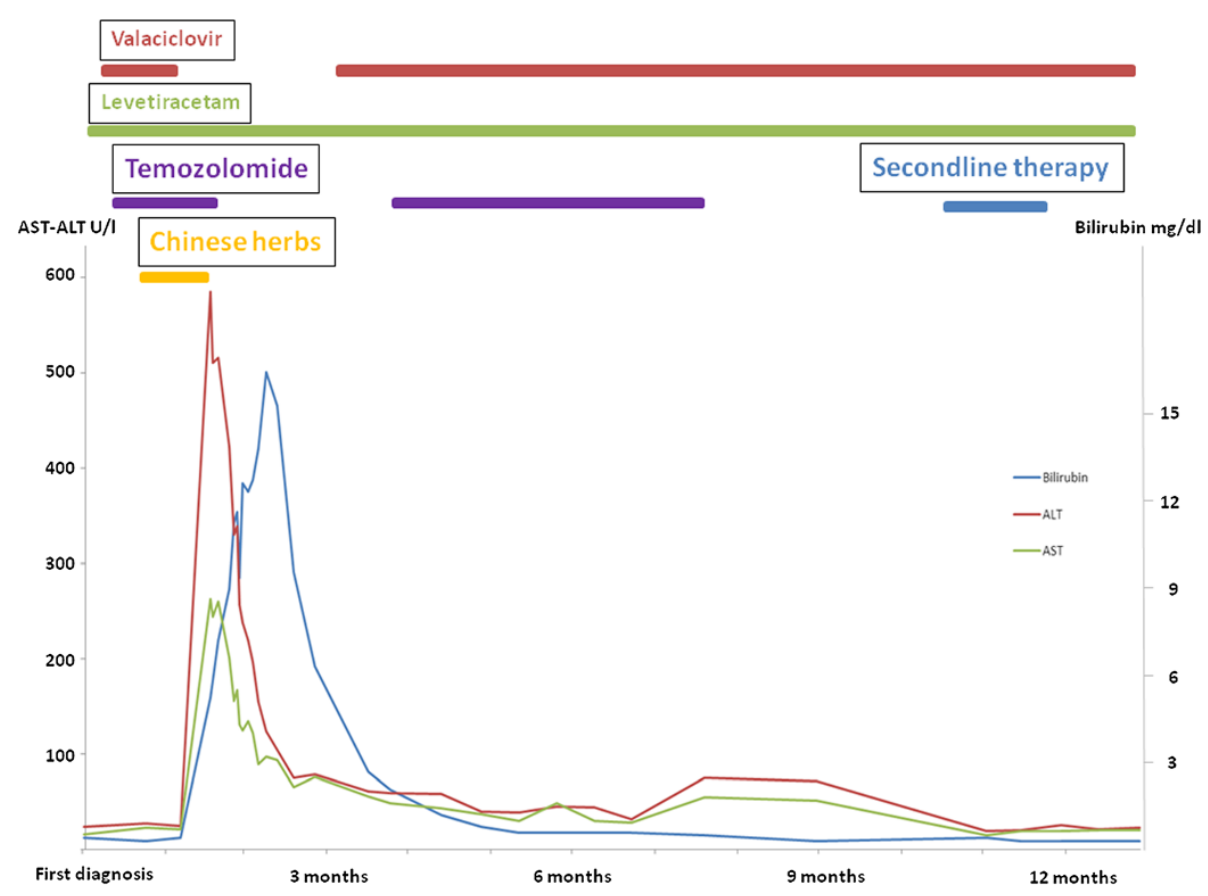

Figure 1 Course of liver enzymes during primary radiochemotherapy with temozolomide and re-exposition for 5 cycles after first diagnosis of glioblastoma.

well as autoantibody screening to exclude an autoimmune hepatitis. Endoscopic retrograde endoscopy excluded pathology of the biliary or pancreatic ductal system. A negative herpes polymerase chain reaction ruled out systemic herpes infection and levetiracetam was reduced in the absence of seizures with the intention to reduce co-medications.

After repeated and detailed conversations the patient reported the use of Chinese herbs as a decoction twice daily for 2 weeks between the beginning of the RCT and the diagnosis of increasing liver enzymes. Following the diagnosis of glioblastoma a general practitioner specialized in CAM recommended the use of a modified form of the Chinese herbal formula called Bu Zhong Yi Qi Wan (11 different herbs) compiled by a local pharmacy, which is commonly used in Chinese CAM. Four weeks after cessation of TEM and the Chinese herbs the liver enzymes started to decrease, but it took about 3 months to normalization of the bilirubin level (Figure 1).

Unfortunately, a [18 F]-fluorethylenthyrosin positron emission tomography positive residual tumor was detected 2 months after the end of radiotherapy. Therefore, despite normalization of the liver parameters was still pending we decided to restart TEM after repeated briefing of the patient to omit any CAM, because we were convinced that this toxic hepatitis was primarily caused by the addition of this Chinese herbal formula. Thus, TEM with an absolute dose of $250 \mathrm{mg}$ per day for 5 days every 4 weeks was re-introduced, supportive medication with valaciclovir and $500 \mathrm{mg}$ bi-daily levetiracetam remained unchanged. We continued treatment for 5 cycles of TEM without any increase of the liver parameters or any other toxicity except for mild cytopenia. Thereafter the patient did not want to continue treatment despite response, because she desired to undergo further complementary treatment. Unfortunately, 3 months after cessation of therapy symptomatic progression due to large edema was diagnosed and combination treatment of bevacizumab and irinotecan was initiated. No liver toxicity or any other severe side effect was observed after 3 months of treatment.

Table 1 Modified form of the Chinese herbal formula called Bu Zhong Yi Qi Wan

\begin{tabular}{ll}
\hline Chinese name & Name and species \\
\hline Dang Shen & Radix Codonopsitis \\
Zhi Gan Cao & Radix Glycyrrhizae \\
Bai Zhun & Rhizoma Atractylodes macrocephala \\
Huang Qi & Radix Astragali \\
Chen Pi & Citrus reticulata \\
Chai Hu & Radix Bupleuri \\
Sheng Ma & Rhizoma Cimicifugae \\
Dang Gui & Radix Angelicae sinensis \\
Ge Gen & Radix Puerariae \\
Du Zhong & Cortex eucommiae \\
Huang Qin & Radix Scutellariae \\
\hline
\end{tabular}




\section{Conclusion}

Liver toxicity is a known but very rare side effect of TEM [2]. Reexpositions to TEM in our patient showed that toxic hepatitis was not causally related to TEM treatment alone or caused by the combination with valaciclovir or levetiracetam. Thus, we conclude that the observed liver toxicity was caused by the concomitant intake of the popular Chinese herbal formula, Bu Zhong Yi Qi Wan, in combination with TEM. Some direct hepatotoxicity by the herbal formula itself seems unlikely, because in traditional Chinese medicine Bu Zhong Yi Qi Wan is thought to be protective to the liver, but cannot be completely ruled out. To exclude an unknown toxic product in the formula we asked for some remnants of this formula, but unfortunately it was totally used up by the patient. Furthermore, the Chinese herbs were recommended by a physician not involved in the management of the patient and probable not familiar with GBM. Due to that we were primarily not aware of this possible interaction and this delayed clarification of this case.

This herbal formula consists of several different herbs and the exact composition varies frequently in CAM. The detailed list of herbs taken by our patient is shown in Table 1 and comprises at least two herbs Huang Qi and Huang Qin with relevant cytochrome $\mathrm{P}_{450} 3 \mathrm{~A} 4$ (CYP3A4) inhibition [3,4]. The effect and safety of temozolomide was not addressed in patients with severe hepatic impairment in clinical trials, but no dose adjustment is recommended in these patients according to manufacturer's labeling. TEM is nonenzymatically converted to the active alkylating metabolite in all tissues and excreted with the urine, therefore CYP isoenzymes are thought to play a minor role up to now. Nevertheless, the distribution of single nucleotide polymorphisms of the CYP450 system is different in Chinese compared to other populations [5]. This would in part explain, why such an interaction is observed in Europe but not in China so far despite integration of TEM in the national insurance schedule in China [6]. Furthermore, Chinese medicine comprises hundreds if not thousands of preparations. It may well slip the attention of physicians if a broad background of intake is assumed.

Furthermore, liver toxicity due to pantoprazol or mirtazapine seems unlikely, because they were started 6 weeks before the start of RCTX without any toxicity and the overall low incidence of liver toxicity of these drugs.

Nevertheless, while the exact mechanism remains unclear, we still observed a severe liver toxicity with the combination of TEM and a popular Chinese formula, which is not reported up to now in the literature. In face of the frequent use of CAM and Chinese herbs in patients with malignant glioma we want to alert cancer physicians about this possible side effect.
We hope to inspire the discussion about the safety of any herbal formulas in combination with cytotoxic therapies and encourage physicians to seek faithful conversations dealing with the use of CAM in cancer patients. There are several reports on the detrimental effects of CAM on the efficacy of chemotherapeutic agents such as St. John's wort with irinotecan or imatinib [7] or ascorbic acid and green tea with bortezomib $[8,9]$ to name but a few. Therefore, we think that a comprehensive management of our patients should also include education about the risk of interactions with herbal formulas.

\section{Consent}

We state that patient's privacy is not compromised by this report and informed consent for publication was obtained.

\section{Competing interests}

T.Me. and L.W. received travel support from Roche. R.G. and A.E. received speaking fees, research support and travel support from Roche. All other authors declare that they have no competing interests.

\section{Authors' contributions}

TMe, TMa, RG and AE are primarily responsible for writing the manuscript. All authors treated the patients, wrote and critically revised the manuscript.

\section{Acknowledgment}

We thank the patient for her consent for publication of this case report.

\section{Author details}

${ }^{1}$ Department of Internal Medicine III, Salzburg Cancer Research Institute, Paracelsus Medical University Salzburg, Austria, Müllner-Hauptstrasse 48, Salzburg 5020, Austria. ${ }^{2}$ Department of Internal Medicine I, Paracelsus Medical University Salzburg, Austria, Müllner-Hauptstrasse 48, Salzburg 5020, Austria.

Received: 3 January 2014 Accepted: 27 March 2014

Published: 30 March 2014

\section{References}

1. Heese O, Schmidt M, Nickel S, Berger H, Goldbrunner R, Tonn JC, Bahr O, Steinbach JP, Simon M, Schramm J, Krex D, Schackert G, Reithmeier T, Nikkhah G, Löffler M, Weller M, Westphal M, German Glioma Network: Complementary therapy use in patients with glioma: an observational study. Neurology 2010, 75:2229-2235.

2. Sarganas G, Orzechowski HD, Klimpel A, Thomae M, Kauffmann W, Herbst H, Bronder E, Garbe E: Severe sustained cholestatic hepatitis following temozolomide in a patient with glioblastoma multiforme: case study and review of data from the FDA adverse event reporting system. Neuro Oncol 2012, 14:541-546.

3. Pao LH, Hu OY, Fan HY, Lin CC, Liu LC, Huang PW: Herb-drug interaction of 50 Chinese herbal medicines on CYP3A4 activity in vitro and in vivo. Am J Chin Med 2012, 40:57-73.

4. Ong ES, Len SM, Lee AC, Chui P, Chooi KF: Proteomic analysis of mouse liver for the evaluation of effects of Scutellariae radix by liquid chromatography with tandem mass spectrometry. Rapid Commun Mass Spectrom 2004, 18:2522-2530.

5. Du J, Xing Q, Xu L, Xu M, Shu A, Shi Y, Yu L, Zhang A, Wang L, Wang $H$, Li X, Feng G, He L: Systematic screening for polymorphisms in the CYP3A4 gene in the Chinese population. Pharmacogenomics 2006, 7:831-841.

6. Chang L, Su J, Jia X, Ren H: Treating malignant glioma in Chinese patients: update on temozolomide. Onco Targets Ther 2014, 7:235-244.

7. Meijerman I, Beijnen $\mathrm{JH}$, Schellens $\mathrm{JH}$ : Herb-drug interactions in oncology: focus on mechanisms of induction. Oncologist 2006, 11:742-752. 
8. Golden EB, Lam PY, Kardosh A, Gaffney K, Cadenas E, Louie SG, Petasis NA Chen TC, Schonthal AH: Green tea polyphenols block the anticancer effects of bortezomib and other boronic acid-based proteasome inhibitors. Blood 2009, 113:5927-5937.

9. Perrone G, Hideshima T, Ikeda H, Okawa Y, Calabrese E, Gorgun G, Santo L, Cirstea D, Raje N, Chauhan D, Baccarani M, Cavo M, Anderson KC: Ascorbic acid inhibits antitumor activity of bortezomib in vivo. Leukemia 2009, 23:1679-1686.

doi:10.1186/1472-6882-14-115

Cite this article as: Melchardt et al:: Liver toxicity during temozolomide chemotherapy caused by Chinese herbs. BMC Complementary and

Alternative Medicine 2014 14:115.

\section{Submit your next manuscript to BioMed Central and take full advantage of:}

- Convenient online submission

- Thorough peer review

- No space constraints or color figure charges

- Immediate publication on acceptance

- Inclusion in PubMed, CAS, Scopus and Google Scholar

- Research which is freely available for redistribution 